\title{
Oral Desmopressin in the Management of Adults with Nocturia
}

\author{
Shahram Gooran ${ }^{1}$, Sevil Nasirmohtaram ${ }^{2}$, Mohammad Ghasem Mohseni ${ }^{1}$, Behzad Narouie ${ }^{3^{*}}$ \\ ${ }^{1}$ Department of Urology, Tehran University of Medical Sciences, Tehran, Iran \\ ${ }^{2}$ Department of ENT, Tehran University of Medical Sciences, Tehran, Iran \\ ${ }^{3}$ Department of Clinical Research Development Center, Zahedan University of Medical Sciences, Zahedan, Iran \\ E-mail: ${ }^{*}$ _nnarouie@yahoo.com \\ Received July 20, 2011; revise August 26, 2011; accepted September 2, 2011
}

\begin{abstract}
We investigated the efficacy of oral desmopressin in the treatment of adult nocturia. In an analytical study between 2007-2009 in Zahedan-Iran, Thirty patients $\geq 55$ years with verified nocturia ( $\geq$ two voids/night) were enrolled. Patients with a history of an obstructive cause of nocturia, those with diseases getting worse by the anti-diuretic affect of desmopressin and those with well-defined curable causes (e.g. cystitis) were excluded. Patients received $0.2 \mathrm{mg}$ of oral desmopressin at bed time for a period of 3 weeks. $p<0.05$ was taken as the significant level. All 30 patients enrolled completed the trial. Fourteen (47\%) patients receiving desmopressin had fewer than half the number of nocturnal voids relative to base line $(p<0.001)$. The mean number of nocturnal voids decreased from 4.6 to $2.4(p<0.001)$. Fatigue $(10 \%)$, headache $(3 \%)$ and dizziness $(3 \%)$ were reported. All adverse events were of mild intensity and there were no instances of hyponatremia. Oral desmopressin is an effective treatment in patients with nocturia and is well-tolerated.
\end{abstract}

Keywords: Nocturia, Desmopressin, Arginine Vasopressin

\section{Introduction}

Nocturia is defined as waking at night to void [1]. Although by definition even a single episode of awakening to urinate is nocturia, most clinicians consider nocturia to have significance when a patient voids two or more times nightly [2]. Nocturia is a very common and bothersome symptom [3]. The prevalence of nocturia increases with age and affects quality of life in both men and women [4]. Rising at night to void increases the risk of personal injury, particularly in the elderly where the condition is associated with a greater risk of falls [5]. Nocturia may be due to either polyuria, nocturnal polyuria or low bladder capacity [6]. As the diurnal variation in AVP release is absent in many older subjects [7], nocturnal polyuria, or the over-production of urine at night, is an important cause of nocturia [8], that may be due to age-related changes in the secretion and action of arginine vasopressin (AVP) $[9,10]$. Treatments currently licensed to improve bladder function do not control nocturnal polyuria [11]. Desmopressin (1-deamino-8-D-arginine vasopressin, ddAVP), a synthetic analogue of AVP is used for treatment of adult nocturia [12], and when administered at bed-time, decreases night-time urine production [13]. Effectively treating nocturia with desmopressin has been shown by some others $[11,14,15]$. The primary aim of the present study was to investigate the efficacy of oral desmopressin in a 3-week treatment period, in reduction in the number of voids at night.

\section{Materials and Methods}

In a analytical study, included 30 patients aged $\geq 55$ years between 2007-2009 in Zahedan-Iran, with complaint of two or more episodes of nocturnal voids during night (night is defined as the period between going to bed for sleeping and waking for rising) and nocturnal diuresis $>0.9 \mathrm{ml} / \mathrm{min}$ [5]. Screening included a precise medical history (including other lower urinary tract symptoms, past medical diseases, fluid intake and medications), physical examination (including measuring supine and standing blood pressure), serum electrolytes, renal function tests and urine analysis. The upper and lower urinary tract ultrasonography with careful attention to post-void residual urine volume was also done. Patients with a serum sodium level below the normal range, 
epilepsy, clinically significant renal, hepatic or cardiovascular disease, uncontrolled hypertension, diabetes insipidus, multiple sclerosis, primary polydipsia, genitourinary tract infection, urge incontinence, and untreated BPH (benign prostatic hyperplasia) were excluded. The number of nocturnal voids and nocturnal urine volume were recorded in the questionnaire before commencing the treatment. The patients received $0.2 \mathrm{mg}$ of oral desmopressin (Minirin ${ }^{\circledR}$, Ferring pharmaceuticals, Sweden) at bed-time for three weeks.

The patients were instructed to drink only to satisfy their thirst until 8 hours after taking the tablets and to stop the treatment and inform their doctor if side-affects such as headaches not relieved by acetaminophen, vision problems, faster heart rate, rapid weight gain, nausea and vomiting, confusion or seizures occur. All patients were visited at the end of the first week of treatment to check their serum sodium levels and probable adverse affects. The primary efficacy endpoint was the proportion of patients who had a reduction by more than half in the mean number of nocturnal voids after treatment compared with baseline. Changes in nocturnal urine volume and number of nocturnal voids, serum sodium levels, incidence of adverse events and patient's satisfaction of desmopressin treatment were also assessed. Statistical analysis was performed by SPSS software (version 15) and results were presented using $\mathrm{P}$ values based on the paired T-test. $p<0.05$ was taken as the significant level.

\section{Results}

Of the patients who reported two or more voids per night, 32 were screened and enrolled. Two patients withdrew consent. The study population comprised 26 men (87\%) and 4 women (13\%), with a median age of 63.5 (55 - 78) years. A clinical response defined as fewer than half the mean number of nocturnal voids after treatment compared with baseline, was achieved by 14 (47\%) patients $(p<0.001)$. If a threshold of $\geq 40 \%$ reduction in nocturnal voids was supposed as a response instead of $\geq 50 \%$, $22(73 \%)$ patients obtained a response to desmopression.The mean number (SD) of voids per night reduced from $4.6(0.9)$ at the baseline to $2.4(0.7)$ at the end of treatment period $(p<0.001)$ (Figure 1). And the mean nocturnal urine volume decreased from $640 \pm 107.6 \mathrm{ml}$ to $395.3 \pm 98.8 \mathrm{ml}(p<0.001)$ (Figure 2$)$. There was a reduction of $\geq 20 \%$ in nocturnal urine volume in 27 $(90 \%)$ patients. In all, $3(10 \%)$ fatigue, $1(3 \%)$ dizziness and $1(3 \%)$ headache were reported. All of adverse events were of mild intensity. No instances of serious side-effects were detected. The mean serum sodium level decreased from 138 to $136 \mathrm{mmol} / \mathrm{L}$ during the first week of treatment $(p=0.317)$ (Figure 3$)$. Serum sodium level $<$

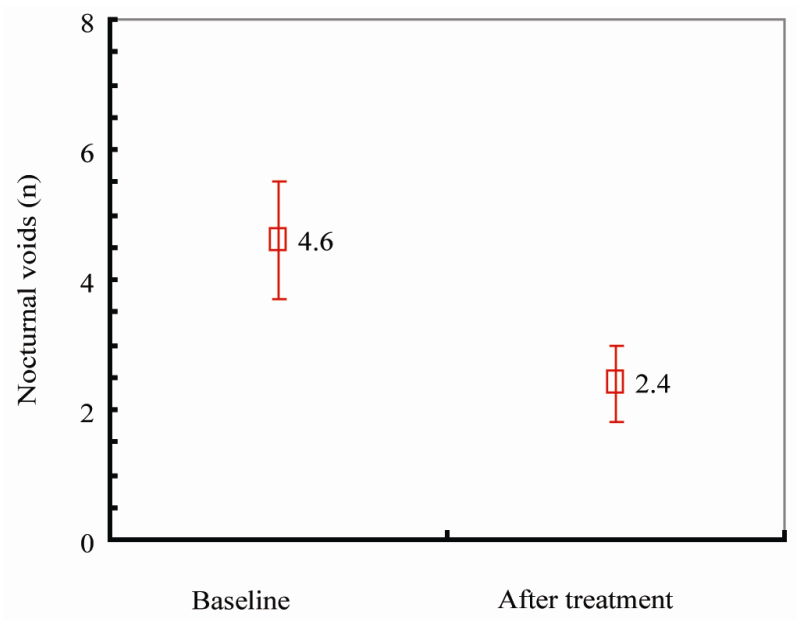

Figure 1. Mean number of nocturnal voids before and after treatment.

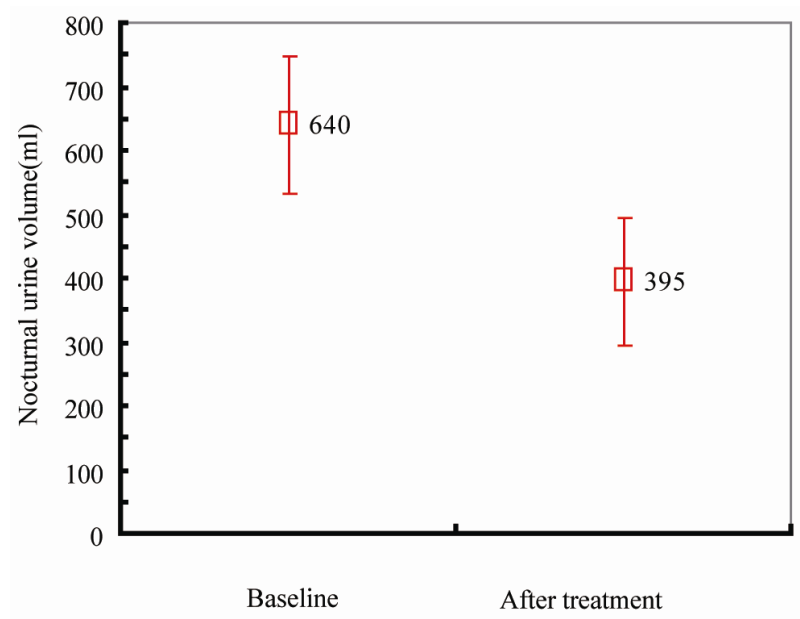

Figure 2. Mean nocturnal urine volume at baseline and after treatment.

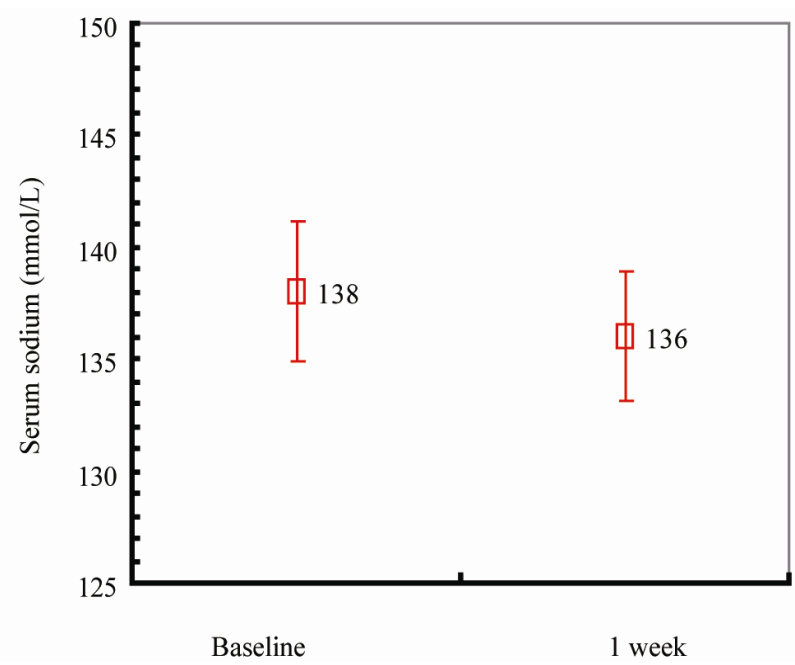

Figure 3. Mean serum sodium values at baseline and 1 week. 
$130 \mathrm{mmol} / \mathrm{L}$ did not occur. Two patients had a decrease of $\geq 5$ units in serum sodium level during the first week of treatment, but both remained asymptomatic during the treatment period and hyponatremia was not reported at all.Twenty-four $(80 \%)$ patients were completely satisfied with the treatment, opposed to $5(17 \%)$ relatively satisfied and $1(3 \%)$ not satisfied.

\section{Discussion}

Adults, particularly the elderly with frequent voiding episodes at night, often suffer from poor sleep and consequently they are often tired during the day, resulting in a poor quality of life [16]. In older patients with nocturia, the day: night urinary output ratio is reduced, and in such patients there is an increased frequency of nocturnal micturition. Plasma vasopressin is at undetectable levels in many such patients [10], therefore desmopressin [a synthetic analogue of arginine vasopressin) seems to be a suitable therapeutic choice for patients with nocturia. As found in previous studies, [13,15,17] desmopressin treatment is associated with a decrease in nocturnal urine output. The main aim of the present study was to investigate the efficacy of oral desmopressin in reduction in episodes of nocturnal voids, which is the main complaint of subjects with nocturia.

The threshold chosen for clinical response was a $\geq$ $50 \%$ reduction in nocturnal void based on previous studies $[11,18]$. The mean number of nocturnal voids reduced by $48 \%$ in patients on desmopressin compared with baseline. In accordance with previous studies, $[14,15,19]$ the decrease in nocturnal diuresis with desmopressin treatment was associated with reduction in nightly voids.

A slight decrease in serum sodium levels during treatment was seen, which was not statistically significant $(p=0.317)$. The study showed that adverse-effects associated with desmopressin treatment were of mild intensity and infrequent, similar to what was previously reported [11]. Caution is warranted in interpreting the results as there was no placebo arm. However, the results accord with those in other trials of desmopressin for this indication. A potential source of efficacy bias may be the safety instructions given to patients, to drink only when thirsty during the night, which could affect fluid intake and thus urine volume, and the number of nocturnal voids. In summary, oral desmopressin given at bed time in patients with nocturia, reduces nocturnal diuresis and nocturnal voids compared with the baseline. As desmopressin is a potential therapeutic choice for subjects with nocturia, patients not responding to general advice and conventional treatments, and those who consider this suffer as a normal consequence of aging, must be informed that it is unnecessary to bear this problem any more. The present study showed desmopressin to have beneficial effects on nocturia and is well-tolerated; however, serum sodium testing is suggested in all patients before and after a few days on treatment.

\section{Acknowledgements}

We would like to acknowledge the Clinical Research Development Center of Ali-ebne-abitaleb Hospital, Zahedan University of Medical Sciences, Zahedan, Iran and Department of Urology, Tehran University of Medical Sciences, School of Medicine, Sina hospital, Tehran, Iran for their help in preparing this manuscript.

\section{References}

[1] P. van Kerrebroeck, P. Abrams, D. Chaikin, et al., "Standardisation Sub-Committee of the International Continence Society. The Standardisation of Terminology in Nocturia: Report from the Standardisation Sub-Committee of the International Continence Society," Neurourology and Urodynamics, Vol. 21, No. 2, 2002, pp. 179-183.

doi:10.1002/nau.10053

[2] F. Y. Chen, Y. T. Dai, C. K. Liu, et al., "Perception of Nocturia and Medical Consulting Behavior among community-Dwelling Women," International Urogynecology Journal and Pelvic Floor Dysfunction, Vol. 18, No. 4, 2007, pp. 431-436. doi:10.1007/s00192-006-0167-x

[3] R. Lundgren, "Nocturia: A New Perspective on an Old Symptom," Scandinavian Journal of Urology and $\mathrm{Ne}$ phrology, Vol. 38, No. 2, 2004, pp. 112-116. doi: $10.1080 / 00365590310020033$

[4] Y. Homma, "Classification of Nocturia in the Adult and Elderly Patient: A Review of Clinical Criteria and Selected Literature," BJU International, Vol. 96, Supplement 1, 2005, pp. 8-14. doi:10.1111/j.1464-410X.2005.05655.x

[5] R. B. Stewart, M. More, F. E. May, et al., "Nocturia a Risk Factor for Falls in the Elderly," Journal of the American Geriatrics Society, Vol. 40, No. 12, 1992, pp. 1217-1220.

[6] S. P. Marinkovic, L. M. Gillen and S. L. Stanton, "Managing nocturia," British Medical Journal, Vol. 328, No. 7447, 2004, pp. 1063-1066.

doi:10.1136/bmj.328.7447.1063

[7] R. Asplund and H. Aberg, "Diurnal Variation in the Levels of Antidiuretic Hormone in the Elderly," Journal of Internal Medicine, 1991, Vol. 229, No. 2, pp. 131-134. doi:10.1111/j.1365-2796.1991.tb00320.x

[8] R. Asplund, "The Nocturnal Polyuria Syndrome (NPS)," General Pharmacology, Vol. 26, No. 6, 1995, pp. 12031209. doi:10.1016/0306-3623(94)00310-J

[9] M. Miller, "Nocturnal Polyuria in Older People: Pathophysiology and Clinical Implications," Journal of the American Geriatrics Society, Vol. 48, No. 10, 2000, pp. 1321-1329.

[10] J. L. Kirkland, M. Lye, D. W. Levy and A. K. Banerjee, 
"Patterns of Urine Flow and Electrolyte Excretion in Healthy Elderly People," British Medical Journal (Clinical Research Ed.), Vol. 287, No. 6406, 1983, pp. 1665-1667. doi:10.1136/bmj.287.6406.1665

[11] A. Mattiasson, P. Abrams, P. Van Kerrebroeck, et al., "Efficacy of Desmopressin in the Treatment of Nocturia: A Double-Blind Placebo-Controlled Study in Men," BJU International, Vol. 89, No. 9, 2002, pp. 855-862. doi:10.1046/j.1464-410X.2002.02791.X

[12] S. Hunskaar, "Epidemiology of Nocturia," BJU International, Vol. 96, Supplement 1, 2005, pp. 4-7. doi:10.1111/j.1464-410X.2005.05650.x

[13] H. Vilhardt, "Basic Pharmacology of Desmopressin: A Review," Drug Investigation, Vol. 2, Supplement 5, 1990, pp. 2-8.

[14] R. Asplund and H. Aberg, "Desmopressin in Elderly Subjects with Increased Nocturnal Diuresis. A Two-Month Treatment Study," Scandinavian Journal of Urology and Nephrology, Vol. 27, No. 1, 1993, pp. 77-82. doi:10.3109/00365599309180419

[15] R. Asplund, B. Sundberg and P. Bengtsson, "Oral Desmopressin for Nocturnal Polyuria in Elderly Subjects: A
Double-Blind, Placebo-Controlled Randomized Exploratory Study," BJU International, Vol. 83, No. 6, 1999, pp. 591-595. doi:10.1046/j.1464-410x.1999.00012.x

[16] R. Asplund and H. Aberg, "Health of the elderly with regard to sleep and nocturnal micturition," Scandinavian Journal of Primary Health Care, Vol. 10, No. 2, 1992, pp. 98-104. doi:10.3109/02813439209014044

[17] S. B. Dimson, "Desmopressin as a Treatment for Enuresis," The Lancet, Vol. 1, No. 8024, 1977, p. 1260.

[18] G. Lose, O. Lalos, R. M. Freeman and P. van Kerrebroeck, "Nocturia Study Group. Efficacy of Desmopressin (Minirin) in the Treatment of Nocturia: A DoubleBlind Placebo-Controlled Study in Women," American Journal of Obstetrics \& Gynecology, Vol. 189, No. 4, 2003, pp. 1106-1113. doi:10.1067/S0002-9378(03)00593-3

[19] S. Salvatore, M. Soligo, F. Proietti, S. Citterio, W. Artibani and R. Milani, "Overactive Bladder Syndrome: Considerations in Pharmacotherapy and New Perspectives," European Journal of Obstetrics \& Gynecology and Reproductive Biology, Vol. 120, No. 2, 2005, pp. 129-133. doi:10.1016/j.ejogrb.2004.10.014 